\title{
Fuzzy Reliability Assessment of Systems With Multiple-Dependent Competing Degradation Processes
}

\author{
Yan-Hui Lin, Yan-Fu Li, Senior Member, IEEE, and Enrico Zio, Senior Member, IEEE
}

\begin{abstract}
Components are often subject to multiple competing degradation processes. For multicomponent systems, the degradation dependence within one component or/and among components need to be considered. Physics-based models and multistate models are often used for component degradation processes, particularly when statistical data are limited. In this paper, we treat dependence between degradation processes within a piecewise-deterministic Markov process (PDMP) modeling framework. Epistemic (subjective) uncertainty can arise due to the incomplete or imprecise knowledge about the degradation processes and the governing parameters, to take this into account, we describe the parameters of the PDMP model as fuzzy numbers. Then, we extend the finitevolume method to quantify the (fuzzy) reliability of the system. The proposed method is tested on one subsystem of the residual heat removal system of a nuclear power plant, and a comparison is offered with a Monte Carlo simulation solution the results show that our method can be most efficient.
\end{abstract}

Index Terms-Epistemic uncertainty, finite-volume (FV) method, fuzzy reliability, fuzzy set theory, multiple-dependent competing degradation processes, piecewise-deterministic Markov process (PDMP).

\section{INTRODUCTION}

I NDUSTRIAL components are often subject to multiple competing degradation processes, whereby any of them may cause failure [1]. For multicomponent systems, the dependence between degradation processes within one component (e.g., the wear of rubbing surfaces influenced by the environmental stress shock within a microengine [2]), or/and the degradation dependence among components (e.g., the degradation of the prefiltrations stations leading to a lower performance level of the sand filter in a water treatment plant [3]) need to be considered.

Physics-based models (PBMs) [4]-[7] and multistate models (MSMs) [8]-[11] are two modeling frameworks that can be used for describing the evolution of degradation in struc-

Manuscript received February 17, 2014; revised June 26, 2014; accepted September 1, 2014. Date of publication October 8, 2014; date of current version October 2, 2015. This work was supported by the Electricité de France Contract 8610-5910112096.

Y.-H. Lin and Y.-F. Li are with the Chair on Systems Science and the Energetic Challenge, European Foundation for New Energy-Electricite' de France, 91192 Gif-sur-Yvette, France, and also with the Department of Industrial Engineering, École Centrale Paris, 92290 Châtenay-Malabry, France (e-mail: yanhui.lin@ ecp.fr; yanfu.li@ecp.fr).

E. Zio is with the Chair on Systems Science and the Energetic Challenge, European Foundation for New Energy-Electricite' de France, 91192 Gif-surYvette, France, with Department of Industrial Engineering, Ecole Centrale Paris, 92290 Châtenay-Malabry, France, and also with the Politecnico di Milano, 20133 Milano, Italy (e-mail: enrico.zio@ecp.fr). tures and components. The former uses physics knowledge that is implemented into mathematical equations for an integrated mechanistic description of the component behavior given the underlying degradation mechanisms (e.g., shocks, fatigue, wear, corrosion, etc.). The latter generally uses degradation and/or failure data from historical field collection or degradation tests, or material science knowledge (e.g., multistate physics model [12]) to describe the degradation processes by a finite number of states of degradation severity and a set of transition rates (estimated from historical data) between the different degradation states.

To treat degradation dependence in a system whose components are modeled by these two types of models, a piecewisedeterministic Markov process (PDMP) approach was employed in our previous work [13]. Monte Carlo (MC) simulation methods [14], [15] can be used to solve PDMP, since the analytical solution is difficult to obtain due to the complex behavior of the system, resulting in the stochasticities of MSMs and time-dependent evolutions of PBMs. However, the major shortcoming is that MC can be quiet time consuming [16]. The finitevolume (FV) scheme studied by Cocozza-Thivent et al. [17] and Eymard et al. [18] appears to be more efficient, leading to comparable results as MC simulation with acceptable computing time [16].

Epistemic (subjective) uncertainty [19] can affect the analysis due to the incomplete or imprecise knowledge about the degradation processes of the components [20], [21]. For PBMs, the parameters (e.g., wear coefficient) and influencing factors (e.g., temperature and pressure) may be unknown [22] and elicited from expert judgment [23], for MSMs, the state performances may be poorly defined due to the imprecise discretization of the underlying continuous degradation processes [24], and the transition rates between states may be difficult to estimate statistically due to insufficient data, especially for those highly reliable critical components (e.g., valves and pumps in nuclear power plants or aircrafts, etc.) [25].

In the literature, fuzzy reliability has been studied by many researchers to account for imprecision and uncertainty in the system model parameters. Tanaka et al. [26] have proposed the fuzzy fault tree for the fuzzy reliability assessment of binarystate systems and Singer [27] has assigned fuzzy probabilities to the basic events. Dunyak et al. [28] have proposed another fuzzy extension to assign fuzzy probability to all events, which is consistent with the calculations from fuzzy fault trees. Ding et al. [20] have developed fuzzy multistate systems (FMSS) models by considering the steady-state probabilities or/and steady-state 
performance levels of a component as fuzzy numbers (FNs). Ding and Lisnianski [29] have proposed the fuzzy universal generating function for the quantification of the fuzzy reliability of FMSS. Later, Li et al. [30] have developed a random fuzzy extension of the universal generating function and Sallak et al. [31] have employed Dempster-Shafer theory to quantify the fuzzy reliability of MSS. Liu et al. [24] have proposed a fuzzy Markov model with fuzzy transition rates for FMSS when the steady fuzzy state probabilities are not available. To the authors' knowledge, none of the previous studies has considered epistemic uncertainty in PDMP system models.

The contributions of the paper are twofold. First, we employ FNs to represent various epistemic uncertainties in multipledependent competing degradation processes modeled by PDMP. Second, we extend the FV scheme for the quantification of PDMP under epistemic uncertainty instead of using timeconsuming MC simulation methods [32], [33]. The reminder of the paper is structured as follows. Section II introduces the PDMP for multiple dependent competing degradation processes. Section III presents the FV scheme for PDMP. Section IV presents the PDMP under uncertainty and the extended FV scheme for system reliability quantification. Section $\mathrm{V}$ presents a case study on one subsystem of the residual heat removal system (RHRS) [34] of a nuclear power plant. Section VI presents numerical results and analysis. Section VII concludes the work.

\section{PieCewise-Deterministic Markov Process FOR SYSTEMS DEGRADATION CONSIDERING DEPENDENCE}

The following assumptions are made on the multiple dependent competing degradation processes of a system [13].

1) The system consists of two groups of components: the first group contains $M$ components, $\vec{L}=\left(L_{1}, L_{2}, \ldots, L_{M}\right)$, whose degradation processes are modeled by PBMs; the second group contains $N$ components $\vec{K}=$ $\left(K_{1}, K_{2}, \ldots, K_{N}\right)$, whose degradation processes are modeled by MSMs including MSPM.

2) All degradation processes of the system follow the PDMP, taking into account the degradation dependence of components within each group and between the groups.

3) For a generic component $L_{m}, m=1,2, \ldots, M$, of the first group, $d_{L m}$ time-dependent continuous variables are used to describe the degradation process, the variables vector ${\overrightarrow{X_{L}}}_{m}(t)=\left({\overrightarrow{X_{L}}}_{m}^{D}(t),{\overrightarrow{X_{L}}}_{m}^{P}(t)\right)$ contains (1) nondecreasing degradation variables ${\overrightarrow{X_{L}}}_{m}(t)$ (e.g., crack length) and (2) physical variables $\vec{X}_{L_{m}} P(t)$ (e.g., velocity and force), whose evolution in time is described by a set of first-order differential equations mathematically representing the underlying physical processes. The component $L_{m}$ fails when one variable of the first type $x_{L_{m}}^{i}(t) \in{\overrightarrow{X_{L}}}_{m}^{D}(t)$ reaches or exceeds its corresponding failure threshold denoted by $x^{i} L_{m}^{*}$, the set of failure states of $L_{m}$ is denoted by $\mathcal{F}_{L m}$.

4) For a generic component $K_{n}, n=1,2, \ldots, N$, in the second group, its discrete degradation state space is denoted by $S_{K_{n}}=\left\{0_{K_{n}}, 1_{K_{n}}, \ldots, d_{K_{n}}\right\}$, ranging from perfect functioning state " $d_{K_{n}}$ " to complete failure state " 0 ." The component is functioning or partially functioning in all generic intermediate states. The transition rates between two different degradation states are used to describe the speed of reaching another degradation state. The performance level of one component (e.g., vibration of the valve due to degradation) at each degradation state and the impact on the other components are considered as deterministic. The failure state set of $K_{n}$ is denoted by $\mathcal{F}_{K_{n}}=\left\{0_{K_{n}}\right\}$.

The degradation condition of the whole system is, then, represented as follows:

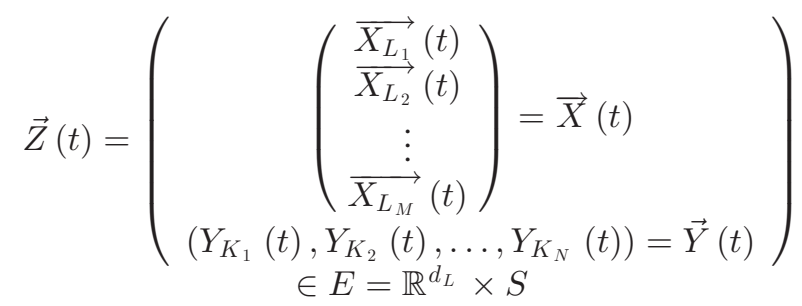

where $Y_{K_{n}}(t), n=1,2, \ldots, N$ denotes the degradation state of component $K_{n}$ at time $t, E$ is a hybrid space of $\mathbb{R}^{d_{L}}\left(d_{L}=\right.$ $\left.d_{L_{1}}+d_{L_{2}}+\cdots+d_{L_{M}}\right)$, and $S\left(S=S_{K_{1}} \times S_{K_{2}} \cdots \times S_{K_{N}}\right)$.

The evolution of the degradation processes $\vec{Z}(t)$ involves the stochastic behavior of $\vec{Y}(t)$ and the deterministic behavior of $\vec{X}(t)$, between two consecutive jumps of $\vec{Y}(t)$, given $\vec{Y}(t)$. Let $\overrightarrow{Y_{k}} \in S, k \in \mathbb{N}$ denote the state of the $N$ components in the second group after $k$ transitions (a transition occurs as long as any one of the $N$ components changes its state), and $T_{k} \in \mathbb{R}^{+}, k \in \mathbb{N}$ denote the time of arrival at state $\overrightarrow{Y_{k}} \cdot \vec{Y}(t)$ is written as follows:

$$
\vec{Y}(t)=\overrightarrow{Y_{k}} \quad \forall t \in\left[T_{k}, T_{k+1}[.\right.
$$

The probability that $\vec{Y}(t)$ will step to state $\vec{J}$ from state $\vec{l}$ in the next infinitesimal time interval $\left[T_{n}, T_{n}+\Delta t\right]$ given $(\vec{Z}(t))_{0 \leq t \leq T_{n}}$, is as follows:

$$
\begin{aligned}
P\left[\overrightarrow{Y_{n+1}}\right. & \left.=\vec{j}, T_{n+1} \in\left[T_{n}, T_{n}+\Delta t\right] \mid(\vec{Z}(t))_{0 \leq t \leq T_{n}}, \overrightarrow{\theta_{K}}\right] \\
& =P\left[\overrightarrow{Y_{n+1}}=\vec{j}, T_{n+1} \in\left[T_{n}, T_{n}+\Delta t\right] \mid \vec{Z}\left(T_{n}\right)\right. \\
& \left.=\left(\vec{X}\left(T_{n}\right), \vec{i}\right), \overrightarrow{\theta_{K}}\right] \\
& =\lambda_{\vec{i}}\left(\vec{j}, \vec{X}\left(T_{n}\right) \mid \overrightarrow{\theta_{K}}\right) \Delta t, \forall n \geq 0, \vec{i}, \vec{j} \in S, \vec{i} \neq \vec{j}
\end{aligned}
$$

where $\overrightarrow{\theta_{K}}$ represents the external influencing factors of the components in the second group and the related coefficients to the transition rates, and $\lambda_{\vec{i}}\left(\vec{j}, \vec{X}\left(T_{n}\right) \mid \overrightarrow{\theta_{K}}\right)$ represents the corresponding transition rate. The evolution of $\vec{X}(t)$, when $t \in\left[T_{k}, T_{k+1}[, k \in \mathbb{N}\right.$, is deterministically described by a set 
of differential equations as follows:

$$
\begin{aligned}
& \overrightarrow{\dot{X}}(t)=\left(\begin{array}{c}
\overrightarrow{X_{L_{1}}}(t) \\
\overrightarrow{X_{L_{2}}}(t) \\
\vdots \\
\overrightarrow{X_{L_{M}}}(t)
\end{array}\right)=\left(\begin{array}{c}
\overrightarrow{f_{L_{1}}}\left(\vec{X}(t), t \mid \overrightarrow{\theta_{L_{1}}}\right) \\
\overrightarrow{\overrightarrow{Y_{k}}}\left(\vec{X}(t), t \mid \overrightarrow{\theta_{L_{2}}}\right) \\
\vdots \\
\overrightarrow{f_{L_{2}}}\left(\overrightarrow{Y_{k}}(t), t \mid \overrightarrow{\theta_{L_{M}}}\right)
\end{array}\right) \\
& =\overrightarrow{f_{L}}\left(\vec{X}(t), t \mid \overrightarrow{\theta_{L}}\right)
\end{aligned}
$$

where $\overrightarrow{f_{L_{m}}^{Y_{k}}}, m=1,2, \ldots, M$ are the set of physics equations, given the influence of the degradation state $\overrightarrow{Y_{k}}$ of the second group components and $\overrightarrow{\theta_{L_{m}}}, m=1,2, \ldots, M$ represents the external influencing factors of the component $L_{n}$ and the physical parameters used in the physics equations. Mathematically, the dependence within each group and between two groups is treated in the framework of a PDMP modeling, where the physics equations in the first group, denoted by $\overrightarrow{f_{L}} \overrightarrow{Y_{k}}\left(\vec{X}(t), t \mid \overrightarrow{\theta_{L}}\right)$, are dependent on the states $\left(\overrightarrow{Y_{k}}\right)$ of the components in the second group and the transition rates in the second group, denoted by $\lambda_{\vec{i}}\left(\vec{j}, \vec{X}(t) \mid t \overrightarrow{\theta_{K}}\right)$, are dependent on the evolution of the variables $(\vec{X}(t))$ in the first group.

The reliability of the system at time $t$ is defined as follows:

$$
R(t)=P[\vec{Z}(s) \notin \mathcal{F} \quad \forall s \leq t]
$$

where $\mathcal{F}=\mathcal{F}_{\vec{X}} \times \mathcal{F}_{\vec{Y}} \subsetneq E$ denotes the space of the failure states of $\vec{Z}(t)$, where $\mathcal{F}_{\vec{X}}$ denotes the subspace of the states of $\vec{X}(t)$, and $\mathcal{F} \vec{Y}$ denotes the subspace of the states of $\vec{Y}(t)$. Let $p_{t}\left(\vec{x}, \vec{i} \mid \overrightarrow{\theta_{L}}, \overrightarrow{\theta_{K}}\right), \vec{x} \in \mathbb{R}^{d_{L}}, \vec{i} \in S$ denote the probability density function (PDF) of processes, $(\vec{X}(t), \vec{Y}(t))_{t \geq 0}$ being in state $(\vec{x}, \vec{i})$ at time $t$, which satisfies

$$
\int_{\mathbb{R}^{d} L} \sum_{\vec{i} \in s} p_{t}\left(\vec{x}, \vec{i} \mid \overrightarrow{\theta_{L}}, \overrightarrow{\theta_{K}}\right) d \vec{x}=1 .
$$

The reliability of the system can be calculated as

$$
R(t)=\int_{\vec{x} \notin \mathcal{F}_{\vec{X}}} \sum_{\vec{i} \notin \mathcal{F}_{\vec{Y}}} p_{t}\left(\vec{x}, \vec{i} \mid \overrightarrow{\theta_{L}}, \overrightarrow{\theta_{K}}\right) d \vec{x} .
$$

The PDF $p_{t}\left(\vec{x}, \vec{i} \mid t \overrightarrow{\theta_{L}}, \overrightarrow{\theta_{K}}\right)$ obeys the ChapmanKolmogorov equation [35] as follows:

$$
\begin{aligned}
\frac{\partial}{\partial t} p_{t}\left(\vec{x}, \vec{i} \mid \overrightarrow{\theta_{L}}, \overrightarrow{\theta_{K}}\right)= & \sum_{\vec{j} \neq \vec{i}} \lambda_{\vec{j}}\left(\vec{i}, \vec{x} \mid \overrightarrow{\theta_{K}}\right) p_{t}\left(\vec{x}, \vec{j} \mid \overrightarrow{\theta_{L}}, \overrightarrow{\theta_{K}}\right) \\
& -\lambda_{\vec{i}}\left(\vec{x} \mid \overrightarrow{\theta_{K}}\right) p_{t}\left(\vec{x}, \vec{i} \mid \overrightarrow{\theta_{L}}, \overrightarrow{\theta_{K}}\right) \\
& -\operatorname{div}\left(\overrightarrow{f_{L}^{\vec{i}}}\left(\vec{x}, t \mid \overrightarrow{\theta_{L}}\right) p_{t}\left(\vec{x}, \vec{i} \mid \overrightarrow{\theta_{L}}, \overrightarrow{\theta_{K}}\right)\right)
\end{aligned}
$$

where $\lambda_{\vec{i}}\left(\vec{x} \mid \overrightarrow{\theta_{K}}\right)=\sum_{\vec{j} \neq \vec{i}} \lambda_{\vec{i}}\left(\vec{j}, \vec{x} \mid \overrightarrow{\theta_{K}}\right)$ is the transition rate departing from the state $\vec{i}$. Among the right-hand parts of (8), the first two terms are due to the stochastic behavior of processes $\vec{Y}(t)$, the first term accounts for the transition of processes $\vec{Z}(t)$ into state $(\vec{i}, \vec{x})$, the second term accounts for the transition of processes $\vec{Z}(t)$ out of state $(\vec{i}, \vec{x})$, the last term is due to the deterministic behavior of processes $\vec{X}(t)$, which represents the volume density of the outward flux of the probability field around the point $(\vec{i}, \vec{x})$. Given the initial probability distribution of the system $p_{0}\left(\vec{x}, \vec{i} \mid \overrightarrow{\theta_{L}}, \overrightarrow{\theta_{K}}\right)$, its evolution in time and that of the system reliability can be obtained solving (8) and (7), respectively.

A challenging problem is to calculate the PDF $p_{t}\left(\vec{x}, \vec{i} \mid t \overrightarrow{\theta_{L}}, \overrightarrow{\theta_{K}}\right)$, because the analytical solution is difficult to obtain due to the complex behavior of the processes [14], [15]. MC simulation methods can be applied for such numerical computations, but the major shortcoming is that they are typically time consuming [16]. FV methods is an alternative that can lead to comparable results as MC simulation, but within a more acceptable computing time [16].

\section{Finite-Volume SCHEME FOR PieCEWISE-Deterministic MARKOV PROCESS}

Instead of directly solving the PDF $p_{t}\left(\vec{x}, \vec{i} \mid \overrightarrow{\theta_{L}}, \overrightarrow{\theta_{K}}\right)$ through the Chapman-Kolmogorov (8), an approximate solution can be obtained by the FV scheme by discretizing the state space of the continuous variables and the time space of PDMP. The approximated solution converges toward the accurate solution under certain conditions. Here, we employ an explicit FV scheme to PDMP, developed by Cocozza-Thivent et al. [17].

\section{A. Assumptions}

This approach can be applied under the following assumptions [17]:

1) The transition rates $\lambda_{\vec{i}}\left(\vec{j}, \cdot \mid \overrightarrow{\theta_{K}}\right) \quad \forall \vec{i}, \vec{j} \in S$ are continuous and bounded functions from $\mathbb{R}^{d_{L}}$ to $\mathbb{R}^{+}$.

2) The physics equations $\overrightarrow{f_{L}^{i}}\left(\cdot, \cdot \mid \overrightarrow{\theta_{L}}\right) \quad \forall \vec{i} \in S$ are continuous functions from $\mathbb{R}^{d_{L}} \times \mathbb{R}^{+}$to $\mathbb{R}^{d_{L}}$ and locally Lipschitz continuous.

3) The physics equations $\overrightarrow{f_{L}^{i}}\left(\cdot, t \mid \overrightarrow{\theta_{L}}\right) \quad \forall \vec{i} \in S$ are sublinear, i.e., there are some $V_{1}>0$ and $V_{2}>0$ such that

4) $\forall \vec{x} \in \mathbb{R}^{d_{L}}, t \in \mathbb{R}^{+}\left|\overrightarrow{f_{L}^{i}}\left(\vec{x}, t \mid \overrightarrow{\theta_{L}}\right)\right| \leq V_{1}(|| \vec{x}||+|t|)+$ $V_{2}$.

5) The functions $\operatorname{div}\left(\overrightarrow{f_{L}^{\vec{i}}}\left(\cdot, \cdot \mid \overrightarrow{\theta_{L}}\right)\right) \quad \forall \vec{i} \in S$ are almost everywhere bounded in absolute value by some real value $\mathrm{D}>0$ (independent of $i$ ).

\section{B. Numerical Scheme}

For the ease of notation, first we let $\overrightarrow{g^{i}}(\cdot, \cdot): \mathbb{R}^{d_{L}} \times \mathbb{R} \rightarrow$ $\mathbb{R} y^{d_{L}}$ denote the solution of

$$
\frac{\partial}{\partial t} \overrightarrow{g^{i}}\left(\vec{x}, t \mid \overrightarrow{\theta_{L}}\right)=\overrightarrow{f_{L}^{\vec{i}}}\left(\overrightarrow{g^{i}}\left(\vec{x}, t \mid \overrightarrow{\theta_{L}}\right), t \mid \overrightarrow{\theta_{L}}\right)
$$$$
\forall \vec{i} \in S, \vec{x} \in \mathbb{R}^{d_{L}}, t \in \mathbb{R}
$$ 
with

$$
\overrightarrow{g^{i}}\left(\vec{x}, 0 \mid \overrightarrow{\theta_{L}}\right)=\vec{x}, \forall \vec{i} \in S, \vec{x} \in \mathbb{R}^{d_{L}}
$$

and $\overrightarrow{g^{i}}\left(\vec{x}, t \mid \overrightarrow{\theta_{L}}\right)$ is the result of the deterministic behavior of $\vec{X}(t)$ after time $t$, starting from the point $\vec{x}$, while the processes $\vec{Y}(t)$ hold on state $\vec{i}$.

The state space $\mathbb{R}^{d_{L}}$ of continuous variables $\vec{X}(t)$ is divided into an admissible mesh $\mathcal{M}$, which is a family of measurable subsets of $\mathbb{R}^{d_{L}} \mathcal{M}$ (is a partition of $\mathbb{R}^{d_{L}}$ ) such that [17]:

1) $\cup_{A \in \mathcal{M}} A=\mathbb{R}^{d_{L}}$

2) $\forall A, B \in \mathcal{M}, A \neq B \Rightarrow A \cap B=\emptyset$;

3) $m_{A}=\int_{A} \overrightarrow{d x}>0 \quad \forall A \in \mathcal{M}$, where $m_{A}$ is the volume of grid $A$;

4) $\sup _{A \in \mathcal{M}} \operatorname{diam}(A)<+\infty$, where $\operatorname{diam}(A)=\sup _{\forall \vec{x}, \vec{y} \in A}$ $|\vec{x}-\vec{y}|$.

Additionally, the time space $\mathbb{R}^{+}$is divided into small intervals $\mathbb{R}^{+}=\cup_{n=0,1,2, \ldots}[n \Delta t,(n+1) \Delta t[$ by setting the time step $\Delta t>0$ (the length of each interval).

The numerical scheme aims at giving an approximate value for the PDF $p_{t}\left(\vec{x}, \vec{i} \mid \overrightarrow{\theta_{L}}, \overrightarrow{\theta_{K}}\right)$ on each $\{i\} \times[n \Delta t,(n+1) \Delta t[\times A, \forall \vec{i} \in S, n \in \mathbb{N}, A \in \mathcal{M}$ denoted by $p_{n}\left(A, \vec{i} \mid \overrightarrow{\theta_{L}}, \overrightarrow{\theta_{K}}\right)$, by assuming that

$$
\begin{aligned}
& p_{t}\left(\vec{x}, \vec{i} \mid \overrightarrow{\theta_{L}}, \overrightarrow{\theta_{K}}\right)=p_{n}\left(A, \vec{i} \mid \overrightarrow{\theta_{L}}, \overrightarrow{\theta_{K}}\right) \\
& \forall \vec{i} \in S, \vec{x} \in A, t \in[n \Delta t,(n+1) \Delta t] .
\end{aligned}
$$

Given the initial PDF $p_{0}\left(\vec{x}, \vec{i} \mid \overrightarrow{\theta_{L}}, \overrightarrow{\theta_{K}}\right)$ of the system at time $t=0, p_{0}\left(A, \vec{i} \mid \overrightarrow{\theta_{L}}, \overrightarrow{\theta_{K}}\right) \quad \forall \vec{i} \in S, A \in \mathcal{M}$ can be obtained as

$$
p_{0}\left(A, \vec{i} \mid \overrightarrow{\theta_{L}}, \overrightarrow{\theta_{K}}\right)=\int_{A} p_{0}\left(\vec{x}, \vec{i} \mid \overrightarrow{\theta_{L}}, \overrightarrow{\theta_{K}}\right) \overrightarrow{d x} / m_{A} .
$$

Then, $p_{n+1}\left(A, \vec{i} \mid \overrightarrow{\theta_{L}}, \overrightarrow{\theta_{K}}\right) \quad \forall \vec{i} \in S, A \in \mathcal{M}, n \in \mathbb{N}$ can be calculated considering the deterministic evaluation of $\vec{X}(t)$ and the stochastic evolution of $\vec{Y}(t)$ based on $p_{n}\left(\mathcal{M}, \vec{i} \mid \overrightarrow{\theta_{L}}, \overrightarrow{\theta_{K}}\right)$ by the Chapman-Kolmogorov forward equation [36] as follows:

$$
\begin{aligned}
& p_{n+1}\left(A, \vec{i} \mid \overrightarrow{\theta_{L}}, \overrightarrow{\theta_{K}}\right) \\
& =\frac{1}{1+\Delta t b_{A}^{\vec{i}}} \widehat{p_{n+1}}\left(A, \vec{i} \mid \overrightarrow{\theta_{L}}, \overrightarrow{\theta_{K}}\right) \\
& \quad+\Delta t \sum_{\substack{\vec{j} \in S \\
\vec{j} \neq \vec{i}}} \frac{\overrightarrow{a_{A}^{j i}}}{1+\Delta t b_{A}^{\vec{j}}} \widehat{p_{n+1}}\left(A, \vec{j} \mid \overrightarrow{\theta_{L}}, \overrightarrow{\theta_{K}}\right)
\end{aligned}
$$

where

$$
a_{A}^{\overrightarrow{j i}}=\int_{A} \lambda_{\vec{j}}\left(\vec{i}, \vec{x} \mid \overrightarrow{\theta_{K}}\right) \overrightarrow{d x} / m_{A} \quad \forall \vec{i} \in S, A \in \mathcal{M}
$$

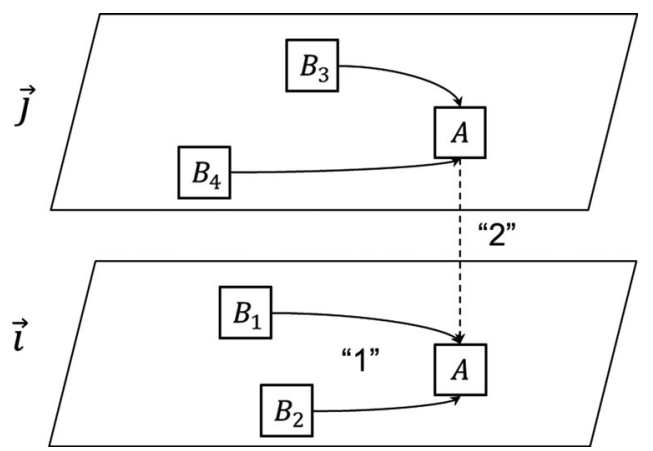

Fig. 1. Evolution of degradation processes during $[n \Delta t,(n+1) \Delta t]$.

is the average transition rate from state $\vec{j}$ to state $\vec{i}$ for grid $A$

$$
b_{A}^{\vec{j}}=\sum_{\vec{i} \neq \vec{j}} a_{A}^{\overrightarrow{j i}} \quad \forall \vec{j} \in S, A \in \mathcal{M}
$$

is the average transition rate out of state $\vec{\iota}$ for grid $A$

$$
\begin{aligned}
\widehat{p_{n+1}}\left(A, \vec{i} \mid \overrightarrow{\theta_{L}}, \overrightarrow{\theta_{K}}\right)=\frac{\sum_{B \in \mathcal{M}} m_{B A}^{\vec{i}} p_{n}\left(B, \vec{i} \mid \overrightarrow{\theta_{L}}, \overrightarrow{\theta_{K}}\right)}{m_{A}} \\
\forall \vec{i} \in S, A \in \mathcal{M}
\end{aligned}
$$

is the approximate value for PDF on $\{i\} \times[(n+1) \Delta t$, $(n+2) \Delta t[\times A$ according to the deterministic evaluation of $\vec{X}(t)$

$$
m_{B A}^{\vec{i}}=\int_{\left\{\vec{y} \in B \mid \overrightarrow{g^{i}}\left(\vec{y}, \Delta t \mid \overrightarrow{\theta_{L}}\right) \in A\right\}} \overrightarrow{d y} \quad \forall \vec{i} \in S, A, B \in \mathcal{M}
$$

is the volume of the part of grid $B$, which will enter grid $A$ after time $\Delta t$ according to the deterministic evaluation of $\vec{X}(t)$.

The first term of the right-hand parts of (13) accounts for the situation that processes $\vec{Y}(t)$ hold on state $\vec{i}$ during time $[n \Delta t,(n+1) \Delta t]$, represented by "1" in an illustrated example in $\mathbb{R}^{2}$ (see Fig. 1), where $\frac{1}{1+\Delta t b_{A}^{\vec{i}}} \quad \forall \vec{i} \in S, A \in \mathcal{M}$ is the approximated probability that no transition happens from state $\vec{i}$ for grid $A$, and the second term of the right-hand parts of (13) accounts for the situation that processes $\vec{Y}(t)$ step to state $\vec{i}$ from another state $\vec{j}$ at time $(n+1) \Delta t$, represented by "2" in an illustrated example in $\mathbb{R}^{2}$ (see Fig. 1), where $a_{A}^{\overrightarrow{j i}} \Delta t \quad \forall \vec{i}, \vec{j} \in S, A \in \mathcal{M}$ is the transition probability from state $\vec{j}$ to state $\vec{\imath}$ for grid $A B_{1}, B_{2}, B_{3}$ and $B_{4}$ [are the grids of which some parts will enter grid $A$ according to the deterministic evaluation of $\vec{X}(t)$ at time $(n+1) \Delta t)]$.

The approximated solution $p_{n}\left(A, \vec{i} \mid \overrightarrow{\theta_{L}}, \overrightarrow{\theta_{K}}\right)$ weakly converges toward the unique solution of (8) when $\Delta t \rightarrow 0$ and $|\mathcal{M}| / \Delta t \rightarrow 0$, where $|\mathcal{M}|=\sup _{A \in \mathcal{M}} \operatorname{diam}(A)$ [17].

\section{Piecewise-Deterministic Markov Process UNDER UNCERTAINTY}

Fuzzy set theories and techniques introduced by Zadeh [37], [38] have been employed in reliability models under epistemic 
uncertainty when the crisp values are insufficient to capture the actual behavior of components. In this study, the following assumptions are made to extend the previous PDMP model with the consideration of epistemic uncertainty.

1) The values of the external influencing factors and physical parameters $\overrightarrow{\theta_{L}}$ in the physics equations $\overrightarrow{f_{L}^{i}}\left(\vec{x}, t \mid \overrightarrow{\theta_{L}}\right)$ $\forall \vec{i} \in S, \vec{x} \in \mathbb{R}^{d_{L}}$ and equations $\overrightarrow{g^{i}}\left(\vec{x}, t \mid \overrightarrow{\theta_{L}}\right) \quad \forall \vec{i} \in$ $S, \vec{x} \in \mathbb{R}^{d_{L}}, t \in \mathbb{R}$ for the deterministic processes $\vec{X}(t)$ can be FNs denoted by $\widetilde{\overrightarrow{\theta_{L}}}$.

2) The values of the external influencing factors and the related coefficients $\overrightarrow{\theta_{K}}$ in the transition rates for the stochastic processes $\vec{Y}(t)$ between two different states $\lambda_{\vec{i}}\left(\vec{j}, \vec{x} \mid \overrightarrow{\theta_{K}}\right) \quad \forall t \in \mathbb{R}^{+}, \vec{x} \in \mathbb{R}^{d_{L}}, \vec{i}, \vec{j} \in S, \vec{i} \neq$ $\vec{j}$ can be FNs denoted by $\widetilde{\overrightarrow{\theta_{K}}}$.

The values of the PDF $p\left(t, \vec{x}, \vec{i} \mid \overrightarrow{\theta_{L}}, \overrightarrow{\theta_{K}}\right)$ and reliability function $R(t)$ therefore, changed from crisp values to FNs denoted by $\tilde{p}\left(t, \vec{x}, \vec{i} \mid \widetilde{\overrightarrow{\theta_{L}}}, \widetilde{\overrightarrow{\theta_{K}}}\right)$ and $\tilde{R}(t)$, respectively. In the next section, we extend the approach presented in Section II to quantify the dependent degradation processes modeled by PDMP under uncertainty.

\section{A. Quantification of Piecewise-Deterministic Markov Process Under Uncertainty}

Let $[\tilde{a}]_{\alpha}=\left[\underline{a}_{\alpha}, \bar{a}_{\alpha}\right]$ denotes the $\alpha$-cut of a fuzzy number $\tilde{a}$, where $\alpha$ and $\bar{a}_{\alpha}$ are the bounds; then, the $\alpha$-cut of $\tilde{p}\left(t, \vec{x}, \vec{i} \mid \widetilde{\overrightarrow{\theta_{L}}}, \overrightarrow{\overrightarrow{\theta_{K}}}\right) \quad \forall \vec{i} \in S, \vec{x} \in \mathbb{R}^{d_{L}}, t \in \mathbb{R}$ can be obtained based on the extension principle [38] as

$$
\begin{aligned}
& {\left[\tilde{p}\left(t, \vec{x}, \vec{i} \mid \widetilde{\overrightarrow{\theta_{L}}}, \widetilde{\overrightarrow{\theta_{K}}}\right)\right]_{\alpha}=}
\end{aligned}
$$

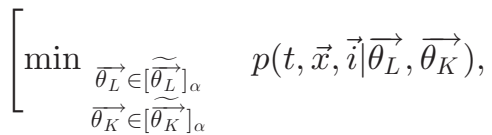

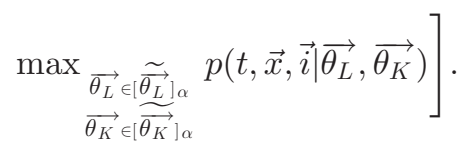

The approximate solution for $\left[\tilde{p}\left(t, \vec{x}, \vec{i} \mid \widetilde{\overrightarrow{\theta_{L}}}, \widetilde{\overrightarrow{\theta_{K}}}\right)\right]_{\alpha} \quad \forall \vec{i} \in$ $S, \vec{x} \in A, t \in\left[n \Delta t,(n+1) \Delta t\left[\right.\right.$ denoted by $\widetilde{p_{n}}\left(A, \vec{i} \mid \widetilde{\overrightarrow{\theta_{L}}}, \widetilde{\overrightarrow{\theta_{K}}}\right)$ can be obtained by varying $\overrightarrow{\theta_{L}}$ in $\left[\overrightarrow{\overrightarrow{\theta_{L}}}\right]_{\alpha}$ and $\overrightarrow{\theta_{K}}$ in $\left[\widetilde{\overrightarrow{\theta_{K}}}\right]_{\alpha}$ as follows:

$$
\begin{aligned}
& \left.\widetilde{p_{n}}\left(A, \vec{i} \mid \widetilde{\overrightarrow{\theta_{L}}}, \widetilde{\overrightarrow{\theta_{K}}}\right)\right]_{\alpha}=
\end{aligned}
$$

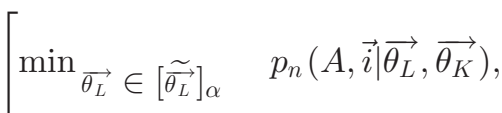

$$
\begin{aligned}
& \overrightarrow{\theta_{K}} \in\left[\widetilde{\overrightarrow{\theta_{K}}}\right]_{\alpha}
\end{aligned}
$$

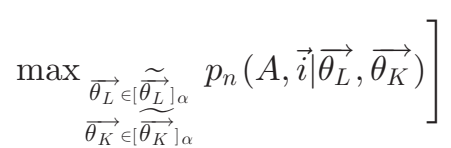

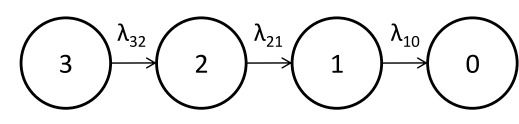

Fig. 2. Degradation processes of the pump.

where $p_{n}\left(A, \vec{i} \mid \overrightarrow{\theta_{L}}, \overrightarrow{\theta_{K}}\right)$ is obtained by (13) through the FV scheme. Then, the parametric programming algorithms [24] can be applied to find the fuzzy probability in (19).

The approximate solution for the $\alpha$-cut of fuzzy reliability $\tilde{R}(t)$ of the system at time $t \in[n \Delta t,(n+1) \Delta t[$ can, then, be obtained as follows:

$$
\begin{aligned}
{[\tilde{R}(t)]_{\alpha}=} & \sum_{A \in \mathcal{M}} \sum_{\vec{i} \notin \mathcal{F}_{\vec{Y}}}\left[\widetilde{p_{n}}\left(A, \vec{i} \mid \widetilde{\overrightarrow{\theta_{L}}}, \widetilde{\overrightarrow{\theta_{K}}}\right)\right]_{\alpha} \\
& \int_{\left\{\vec{x} \in A \mid \vec{x} \notin \mathcal{F}_{\vec{X}}\right\}} \overrightarrow{d x}
\end{aligned}
$$

In most cases, the original $R(t)$ is monotonic with $\overrightarrow{\theta_{L}}$ and $\overrightarrow{\theta_{k}}$; then, we can directly obtain that instead of using (19)

$$
\begin{aligned}
& {[\tilde{R}(t)]_{\alpha}=} \\
& {\left[\sum_{A \in \mathcal{M}} \sum_{\vec{i} \notin \mathcal{F}_{\vec{Y}}} p_{n}\left(A, \vec{i} \mid \overrightarrow{{\underline{\theta_{L}}}_{\alpha}}, \overrightarrow{{\overrightarrow{\theta_{K}}}_{\alpha}}\right) \int_{\left\{\vec{x} \in A \mid \vec{x} \notin \mathcal{F}_{\vec{X}}\right\}} \overrightarrow{d x}\right.} \\
& \left.\sum_{A \in \mathcal{M}} \sum_{\vec{i} \notin \mathcal{F}_{\vec{Y}}} p_{n}\left(A, \vec{i} \mid \overrightarrow{\overrightarrow{\theta_{L}}}, \overrightarrow{\overrightarrow{\theta_{K}}}\right) \int_{\left\{\vec{x} \in A \mid \vec{x} \notin \mathcal{F}_{\vec{X}}\right\}} \overrightarrow{d x}\right] .
\end{aligned}
$$

\section{Illustrative Case}

The illustrative case refers to one important subsystem of a RHRS consisting of a centrifugal pump and a pneumatic valve. The definition of the system has been provided by Électricité de France (EDF). The degradation model of the pump is a modified MSM from the one originally supplied by EDF, while that of the valve is a PBM developed by Daigle and Goebel [4]. Upon discussion with the experts, a degradation dependence between the two components has been considered, as follows. The degradation of the pump will cause it to vibrate [39], which in turn, will lead the valve to vibrate and, therefore, aggravate the degradation processes of the latter [40].

Given its series logic structure, the subsystem is considered failed, when one of the two components is failed.

\section{A. Centrifugal Pump}

The multistate model of the degradation processes of the centrifugal pump is a continuous-time homogeneous Markov chain with constant transition rates as shown in Fig. 2.

There are four degradation states for the pump, from the perfect functioning state " 3 "to the complete failure state " 0. ." Due to the degradation, the pump can vibrate when it reaches the degradation states " 2 " and " 1 ." The intensity of the vibration of the state "2" is assigned as "smooth" and that of the state " 1 " is assigned as "rough" by the experts. Let $Y_{p}(t)$ denotes the 


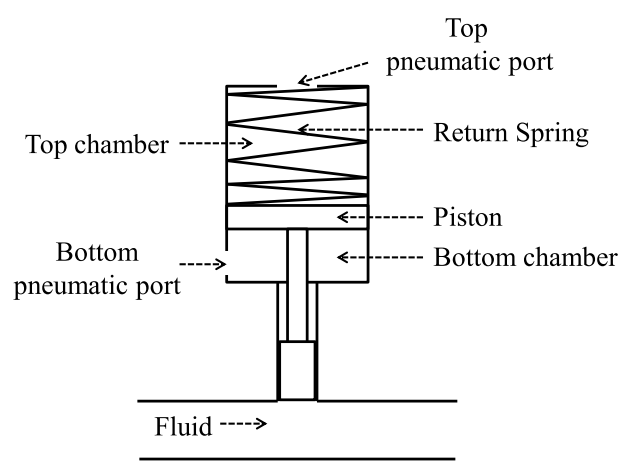

Fig. 3. Simplified scheme of the pneumatic valve [4].

degradation state of the pump at time $t$, and $S_{p}=$ $\left\{{ }^{\prime} 0\right.$ ' ' ' 1 ', '2', ' 3 ' $\}$ denotes the degradation states set. The pump is functioning until it reaches the complete failure state " 0 "; $\lambda_{32}$, $\lambda_{21}$, and $\lambda_{10}$ are the transition rates of the degradation process.

\section{B. Pneumatic Valve}

The simplified scheme of the pneumatic valve is shown in Fig. 3.

The pneumatic valve is a normally closed and gas-actuated valve with a linear cylinder actuator. Top chamber and bottom chamber are separated by the piston, and are connected to a top pneumatic port and a bottom pneumatic port, respectively. The position of the piston between fully closed position " 0 " and fully open position " $x_{s}$ " can be controlled by regulating the pressure of the pneumatic ports to fill or evacuate the two chambers. A return spring is linked with the piston to ensure that the valve will close when pressure is lost, due to the spring force.

There are several common degradation mechanisms of the valve (e.g., sliding wear, internal leaks, external leaks, etc.). In this case study, as a degradation mechanism, we have chosen the external leak at the actuator connections to the bottom pneumatic port due to corrosion and other environmental factors, for two reasons. 1) It is more significant than the other degradation mechanisms according to the results shown in [4]. 2) The uncertainty associated with the wear coefficient estimated from a limited amount of data should be taken into account. The leak will lead the valve to be more difficult to open, but easier to close. The threshold of the area of leak hole $D_{b}^{*}$ is defined as the value above which $\left(D_{b}(t)>D_{b}^{*}\right)$, the valve cannot reach the fully open position within the 15-s time limit from the fully closed position, after an opening command is executed.

Let $D_{b}(t)$ denotes the area of the leak hole at the bottom pneumatic port at time $t$, the development of the leak size is described by

$$
D_{b}(t)=\omega_{b}\left(1+\beta_{Y_{p}(t)}\right)
$$

where $\omega_{b}$ is the original wear coefficient, and where $\beta_{Y_{p}(t)}$ is the relative increment of the developing rate of the external leak at the bottom pneumatic port caused by the vibration of the pump at the degradation state " 2 " or "1" (if we ignore the degradation dependence, then $\beta_{Y_{p}(t)}=0$ ).
The function command of the valve cycle is a 30 -s periodic signal, and the valve is commanded to open in the first half period and to close in the second half by changing the pressure of the top bottom pneumatic port $u_{t}(t)$ and that of the bottom pneumatic port $u_{b}(t)$ (opening command: $u_{t}(t)=P_{\text {atm }}$ and $u_{b}(t)=$ $P_{\text {sup }}$; closing command: $u_{t}(t)=P_{\text {sup }}$ and $\left.u_{b}(t)=P_{\text {atm }}\right)$. At the beginning, the valve is set to the fully closed position.

Let $x(t)$ denotes the position of the valve at time $t$, whose evolution in time is described by the following equations:

$$
\ddot{x}(t)=a(t)
$$

where

$$
\begin{aligned}
a(t)= & \frac{1}{m}\left[\left(p_{b}(t)-p_{t}(t)\right) A_{p}-m g+\right. \\
& \left.-k\left(x(t)+x_{0}\right)-r v(t)+F_{c}(x(t))\right]
\end{aligned}
$$

is the valve acceleration, where

$$
p_{b}(t)=\frac{m_{b}(t) R_{g} T}{V_{b 0}+A_{p} x(t)}
$$

is the gas pressure of the bottom of the piston

$$
p_{t}(t)=\frac{m_{t}(t) R_{g} T}{V_{t 0}+A_{p}\left(x_{s}-x(t)\right)}
$$

is the gas pressure of the top of the piston, and where

$$
\begin{aligned}
m_{t}(t) & =m_{t}(0)+\int_{0}^{t} f_{g}\left(u_{t}(t), p_{t}(t), A_{s}\right) d t \\
\text { with } m_{t}(0) & =\frac{P_{\mathrm{sup}}\left(L_{s} A_{p}+V_{t 0}\right)}{R_{g} T}
\end{aligned}
$$

and

$$
\begin{aligned}
m_{b}(t)= & m_{b}(0)+\int_{0}^{t} f_{g}\left(u_{b}(t), p_{b}(t), A_{s}\right) \\
& +f_{g}\left(P_{\mathrm{atm}}, p_{b}(t), D_{b}(t)\right) d t \\
\text { with } m_{b}(0)= & \frac{P_{\mathrm{atm}} V_{b 0}}{R_{g} T}
\end{aligned}
$$

are respectively the masses of the gas in the top chamber and bottom chamber at time $t$, and where

$$
\begin{aligned}
& f_{g}\left(p_{1}, p_{2}, A\right) \\
& = \begin{cases}\varepsilon P C_{s} A \sqrt{\frac{\gamma}{z R_{g} T}\left(\frac{2}{\gamma+1}\right)^{\frac{\gamma+1}{\gamma-1}}}, & \text { if } \delta \leq\left(\frac{2}{\gamma+1}\right)^{\frac{\gamma}{\gamma-1}} \\
\varepsilon P C_{s} A \sqrt{\frac{\gamma}{z R_{g} T}\left(\frac{2}{\gamma-1}\right)\left(\delta^{\frac{2}{\gamma}}-\delta^{\frac{\gamma+1}{\gamma}}\right)}, & \text { if } \delta>\left(\frac{2}{\gamma+1}\right)^{\frac{\gamma}{\gamma-1}}\end{cases} \\
& \text { with }\left\{\begin{array}{l}
P=\max \left(p_{1}, p_{2}\right) \\
\delta=\frac{\min \left(p_{1}, p_{2}\right)}{\max \left(p_{1}, p_{2}\right)} \\
\varepsilon=\operatorname{sgn}\left(p_{1}-p_{2}\right)
\end{array}\right.
\end{aligned}
$$


TABLE I

PARAMETER DEFINITIONS AND VALUES

\begin{tabular}{|c|c|}
\hline Parameter Definition & Value \\
\hline$g$-acceleration due to gravity & $9.8 \mathrm{~m} / \mathrm{s}$ \\
\hline$P_{\text {sup }}$ - supply pressure & $5.27 \mathrm{e} 6 \mathrm{~Pa}$ \\
\hline$P_{\text {atm }}$-atmospheric pressure & $1.01 \mathrm{e} 5 \mathrm{~Pa}$ \\
\hline$m$-mass of the moving parts of the valve & $50 \mathrm{~kg}$ \\
\hline$r$-coefficient of kinetic friction & $6.00 \mathrm{e} 3 \mathrm{Ns} / \mathrm{m}$ \\
\hline$k$-spring constant & $4.80 \mathrm{e} 4 \mathrm{~N} / \mathrm{s}$ \\
\hline$k_{c}$-large spring constant associated with the flexible seals & $1.00 \mathrm{e} 8 \mathrm{~N} / \mathrm{s}$ \\
\hline$x_{0}$-amount of spring compression when the valve is closed & $0.254 \mathrm{~m}$ \\
\hline$x_{s}$-fully open position of the valve & $0.1 \mathrm{~m}$ \\
\hline$A_{p}$-surface area of the piston & $8.10 \mathrm{e}-3 \mathrm{~m}^{2}$ \\
\hline$V_{t 0}-$ minimum gas volume of the top chamber & $8.11 \mathrm{e}-4 \mathrm{~m}^{3}$ \\
\hline$V_{b 0}-$ minimum gas volume of the bottom chamber & $8.11 \mathrm{e}-4 \mathrm{~m}^{3}$ \\
\hline$R_{\mathrm{g}}$ —gas constant for the pneumatic gas & $296 \mathrm{~J} / \mathrm{K} / \mathrm{kg}$ \\
\hline$T$-ideal gas temperature & $293 \mathrm{~K}$ \\
\hline$\gamma$-ratio of specific heats & 1.4 \\
\hline$z$ - gas compressibility factor & 1 \\
\hline$A_{s}$-orifice area of the pneumatic port & $1.00 \mathrm{e}-5 \mathrm{~m}^{2}$ \\
\hline$C_{s}$-flow coefficient & 0.1 \\
\hline
\end{tabular}

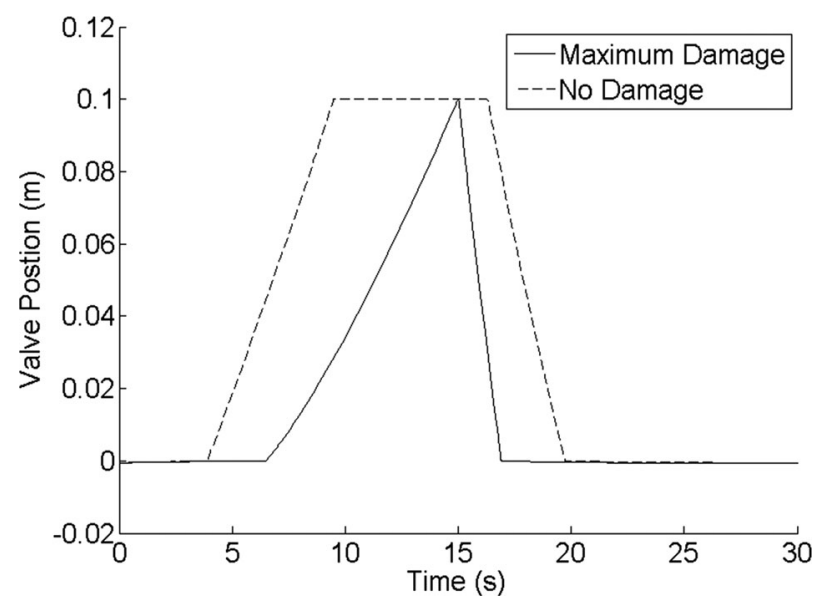

Fig. 4. Valve behavior with different sizes of the external leak.

defines the gas flow through an orifice, and

$$
F_{c}(x(t))= \begin{cases}k_{c}(-x(t)), & \text { if } x(t)<0 \\ 0, & \text { if } 0 \leq x(t) \leq L_{s} \\ -k_{c}\left(x(t)-x_{s}\right), & \text { if } x(t)>L_{s}\end{cases}
$$

is the contact force exerted on the piston by the flexible seals.

The parameters definitions and values (except for $\omega_{b}$ and $\left.\beta_{Y_{p}(t)}\right)$ of the valve are presented in Table I.

With the given values, the threshold of the area of leak hole $D_{b}^{*}=1.06 \mathrm{e}-5 \mathrm{~m}^{2}$ (maximum damage) can be calculated: once exceeded, the valve will not reach the fully open position within the $15 \mathrm{~s}$ limit, as shown in Fig. 4.
TABLE II

VALUES OF THE FUZZY PARAMETERS IN PDMP

\begin{tabular}{lc}
\hline \hline Parameter & Value \\
\hline$\widetilde{\omega_{b}}$ & $(9 \mathrm{e}-9,1 \mathrm{e}-8,1.1 \mathrm{e}-8) \mathrm{m}^{2} / \mathrm{s}$ \\
$\widetilde{\beta_{2}}$ & $(9 \%, 10 \%, 11 \%)$ \\
$\widetilde{\beta_{1}}$ & $(18 \%, 20 \%, 22 \%)$ \\
$\widetilde{\lambda_{32}}$ & $(2.7 \mathrm{e}-3,3 \mathrm{e}-3,3.3 \mathrm{e}-3) \mathrm{s}-1$ \\
$\widetilde{\lambda_{21}}$ & $(2.7 \mathrm{e}-3,3 \mathrm{e}-3,3.3 \mathrm{e}-3) \mathrm{s}-1$ \\
$\widetilde{\lambda_{10}}$ & $(2.7 \mathrm{e}-3,3 \mathrm{e}-3,3.3 \mathrm{e}-3) \mathrm{s}-1$ \\
\hline \hline
\end{tabular}

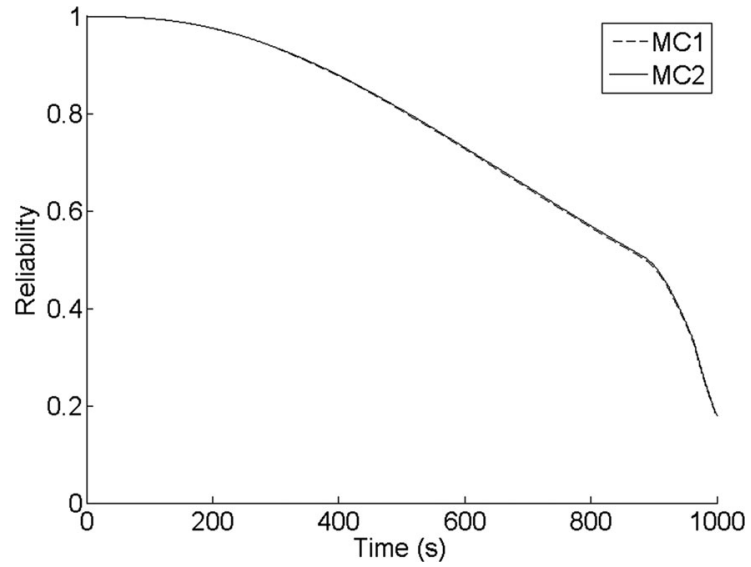

Fig. 5. Fuzzy reliability at cut level $\alpha=1$ (no fuzziness) obtained by MC1 and $\mathrm{MC} 2$.

\section{Piecewise-Deterministic Markov Process for the System Under Uncertainty}

The degradation processes of the whole system are modeled by PDMP as follows:

$$
\vec{Z}(t)=\left(\begin{array}{c}
D_{b}(t) \\
Y_{p}(t)
\end{array}\right) \in \mathbb{R}^{+} \times S_{p}
$$

The space of the failure states of $\vec{Z}(t)$ is $\mathcal{F}=\mathcal{F}_{D_{b}} \times$ $\mathcal{F}_{Y_{p}}=\left[D_{b}^{*},+\infty\right) \times\{0\}$. We have $\overrightarrow{\theta_{L}}=\left(\omega_{b}, \beta_{Y_{p}(t)}\right)$ and $\overrightarrow{\theta_{K}}=$ $\left(\lambda_{32}, \lambda_{21}, \lambda_{10}\right)$, which are the uncertain parameters due to the fact that their values are estimated from insufficient degradation data or elicited from expert judgment. Epistemic uncertainty associated with them, hence, needs to be taken into account, and a proper mathematical representation of uncertainty of this nature is by FNs. We choose triangular fuzzy numbers [41] to represent the uncertain parameters because their boundary values and most probable or most advisable values are considered easier to be elicited from experts than other FN types and they are widely used to represent uncertain parameters in reliability engineering [20], [24], [29], [41]. However, the proposed framework is generally suitable for FNs with other types of membership functions. The values of $\widetilde{\omega_{b}}, \widetilde{\beta_{Y_{p}(t)}}, \widetilde{\lambda_{32}}, \widetilde{\lambda_{21}}$, and $\widetilde{\lambda_{10}}$ are shown in Table II. The FNS are assigned by considering a relative uncertainty of $\pm 10 \%$ of the original parameters values. 


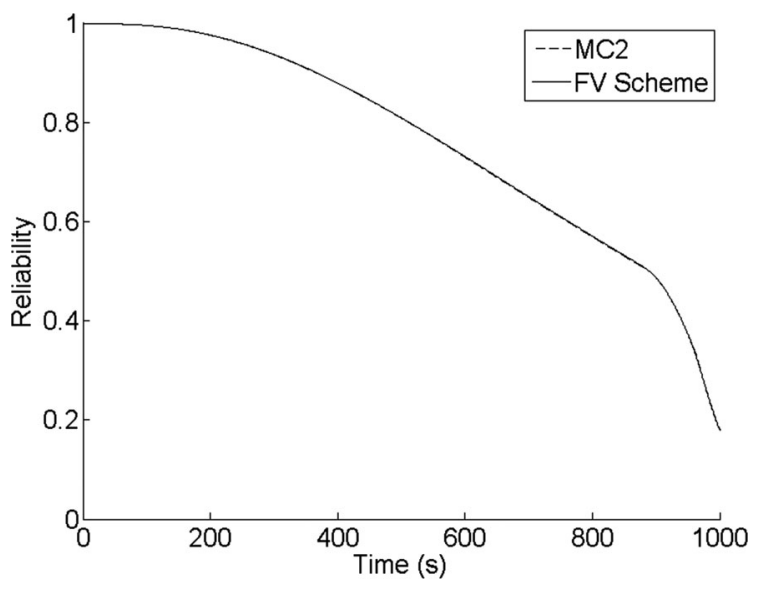

Fig. 6. Fuzzy reliability at cut level $\alpha=1$ (no fuzziness) obtained by MC2 and FV scheme.

TABLE III

VALUES OF THE FUZZY PARAMETERS IN PDMP

\begin{tabular}{lccccc}
\hline \hline Method & MC2 & MC1 & Relative difference & FV scheme & Relative difference \\
\hline Time & & & & & \\
$100 \mathrm{~s}$ & 0.9965 & 0.9966 & $0.01 \%$ & 0.9964 & $-0.01 \%$ \\
$200 \mathrm{~s}$ & 0.9769 & 0.9766 & $-0.03 \%$ & 0.9773 & $0.04 \%$ \\
$300 \mathrm{~s}$ & 0.9372 & 0.9364 & $-0.09 \%$ & 0.9379 & $0.07 \%$ \\
$400 \mathrm{~s}$ & 0.8799 & 0.8780 & $-0.22 \%$ & 0.8805 & $0.07 \%$ \\
$500 \mathrm{~s}$ & 0.8094 & 0.8063 & $-0.38 \%$ & 0.8102 & $0.10 \%$ \\
$600 \mathrm{~s}$ & 0.7305 & 0.7283 & $-0.30 \%$ & 0.7321 & $0.22 \%$ \\
$700 \mathrm{~s}$ & 0.6496 & 0.6469 & $-0.42 \%$ & 0.6513 & $0.26 \%$ \\
$800 \mathrm{~s}$ & 0.5696 & 0.5664 & $-0.56 \%$ & 0.5714 & $0.32 \%$ \\
$900 \mathrm{~s}$ & 0.4873 & 0.4839 & $-0.70 \%$ & 0.4874 & $0.02 \%$ \\
$1000 \mathrm{~s}$ & 0.1801 & 0.1778 & $-1.28 \%$ & 0.1811 & $0.56 \%$ \\
\hline \hline
\end{tabular}

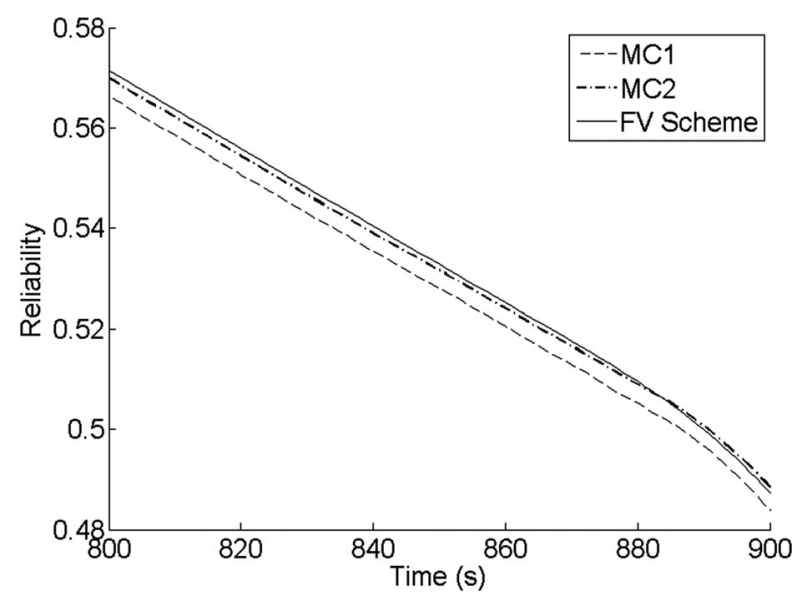

Fig. 7. Fuzzy reliability at cut level $\alpha=1$ (no fuzziness) obtained by MC1, $\mathrm{MC} 2$, and FV scheme of time horizon between 800 and $900 \mathrm{~s}$.

The initial state of the system is assumed as follows:

$$
\overrightarrow{Z_{0}}=\left(\begin{array}{c}
D_{b}(0) \\
Y_{p}(0)
\end{array}\right)=\left(\begin{array}{c}
0 \\
{ }^{\prime} 3
\end{array}\right)
$$

which means that the two components are both in their perfect state. The initial PDF of the processes $\left(D_{b}(t), Y_{p}(t)\right)_{t \geq 0}$,

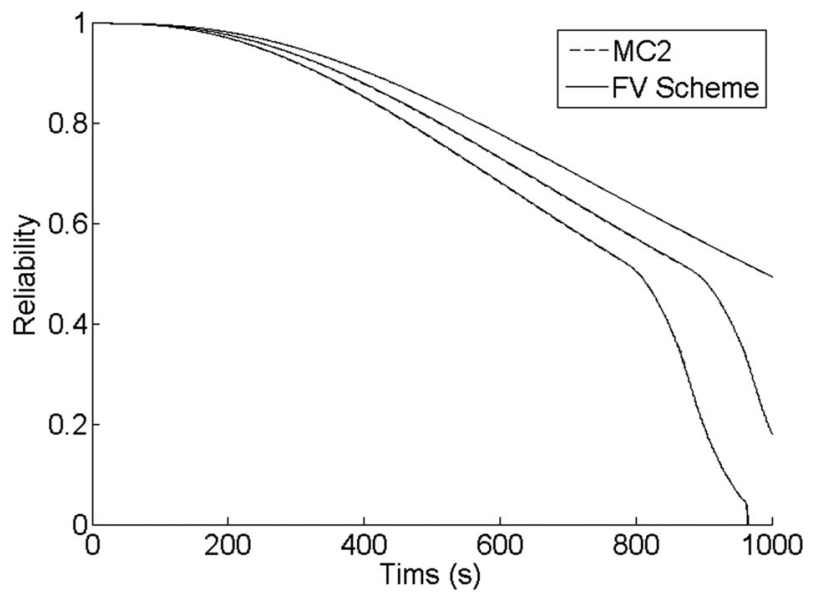

Fig. 8. Fuzzy reliability at cut levels $\alpha=0$ and $\alpha=1$ obtained by MC2 and FV scheme.

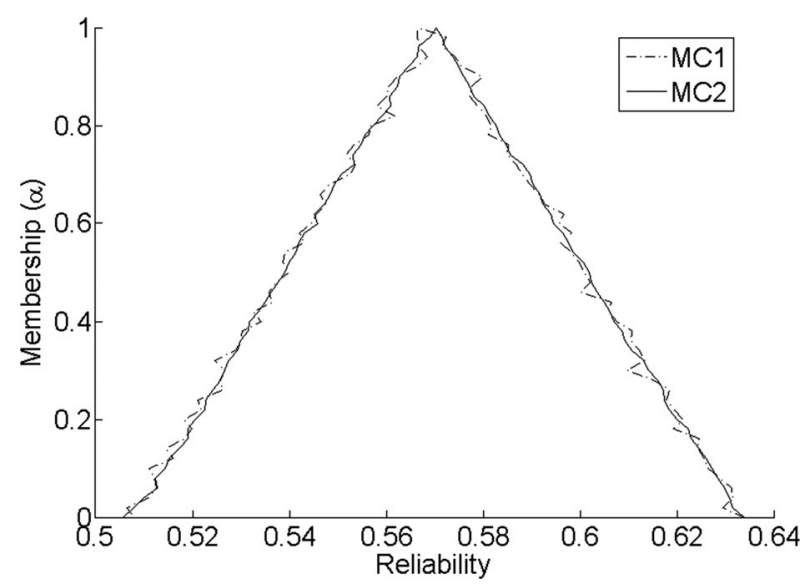

Fig. 9. Membership function of fuzzy reliability $\widetilde{R}(t)$ at mission time $t=$ $800 \mathrm{~s}$ obtained by MC1 and MC2.

$p_{0}\left(x, i \mid \widetilde{\overrightarrow{\theta_{L}}}, \widetilde{\overrightarrow{\theta_{K}}}\right)$, hence, equals to 1 if $(x, i)=(0$, ' 3 ' $)$ and to 0 otherwise.

\section{RESULTS}

A MC-based approach [33] can also be used to quantify the epistemic uncertainty, in alternative to the fuzzy arithmetic operations and fuzzy parameter programming procedure. The comparisons between the results of the reliability of the system at cut level $\alpha=1$, i.e., without fuzziness in the parameters values, over a time horizon $1000 \mathrm{~s}$ calculated by MC simulation and the FV scheme are shown in Figs. 5 and 6. In order to better understand the differences presented in Figs. 5 and 6, we have added below each original figure one extra figure (Fig. 7), zooming on the time horizon between 800 and $900 \mathrm{~s}$ to illustrate the results obtained by different methods. For the FV 
TABLE IV

COMPARISON OF THE RESUlTS OF THE MEMBERSHIP FunCTION OBTAINED BY MC SiMULATION METHODS AND FV SCHEME

\begin{tabular}{|c|c|c|c|c|c|}
\hline Method & $\mathrm{MC} 2$ & MC1 & Relative difference (Minimum/Maximum) & FV scheme & Relative difference (Minimum/Maximum) \\
\hline \multicolumn{6}{|l|}{ Cut level } \\
\hline$\alpha=0$ & {$[0.5062,0.6330]$} & {$[0.5086,0.6340]$} & $0.47 \% / 0.16 \%$ & {$[0.5057,0.6350]$} & $-0.10 \% / 0.32 \%$ \\
\hline$\alpha=0.1$ & {$[0.5137,0.6271]$} & {$[0.5111,0.6260]$} & $-0.51 \% / 0.18 \%$ & {$[0.5148,0.6285]$} & $0.21 \% / 0.22 \%$ \\
\hline$\alpha=0.2$ & {$[0.5209,0.6203]$} & {$[0.5181,0.6218]$} & $-0.54 \% / 0.24 \%$ & {$[0.5220,0.6221]$} & $0.21 \% / 0.29 \%$ \\
\hline$\alpha=0.3$ & {$[0.5266,0.6141]$} & {$[0.5249,0.6095]$} & $-0.32 \% /-0.75 \%$ & {$[0.5283,0.6157]$} & $0.32 \% / 0.26 \%$ \\
\hline$\alpha=0.4$ & {$[0.5329,0.6088]$} & {$[0.5348,0.6071]$} & $0.36 \% /-0.28 \%$ & {$[0.5344,0.6093]$} & $0.28 \% / 0.08 \%$ \\
\hline$\alpha=0.5$ & {$[0.5386,0.6015]$} & {$[0.5413,0.6001]$} & $0.50 \% /-0.23 \%$ & {$[0.5405,0.6030]$} & $0.35 \% / 0.25 \%$ \\
\hline$\alpha=0.6$ & {$[0.5440,0.5955]$} & {$[0.5476,0.5976]$} & $0.66 \% / 0.35 \%$ & {$[0.5466,0.5966]$} & $0.48 \% / 0.18 \%$ \\
\hline$\alpha=0.7$ & {$[0.5513,0.5892]$} & {$[0.5529,0.5880]$} & $0.29 \% /-0.20 \%$ & {$[0.5528,0.5903]$} & $0.27 \% / 0.19 \%$ \\
\hline$\alpha=0.8$ & {$[0.5577,0.5825]$} & {$[0.5559,0.5808]$} & $-0.32 \% /-0.29 \%$ & {$[0.5590,0.5840]$} & $0.23 \% / 0.26 \%$ \\
\hline$\alpha=0.9$ & {$[0.5626,0.5756]$} & {$[0.5643,0.5797]$} & $0.30 \% / 0.71 \%$ & {$[0.5652,0.5777]$} & $0.46 \% / 0.36 \%$ \\
\hline
\end{tabular}

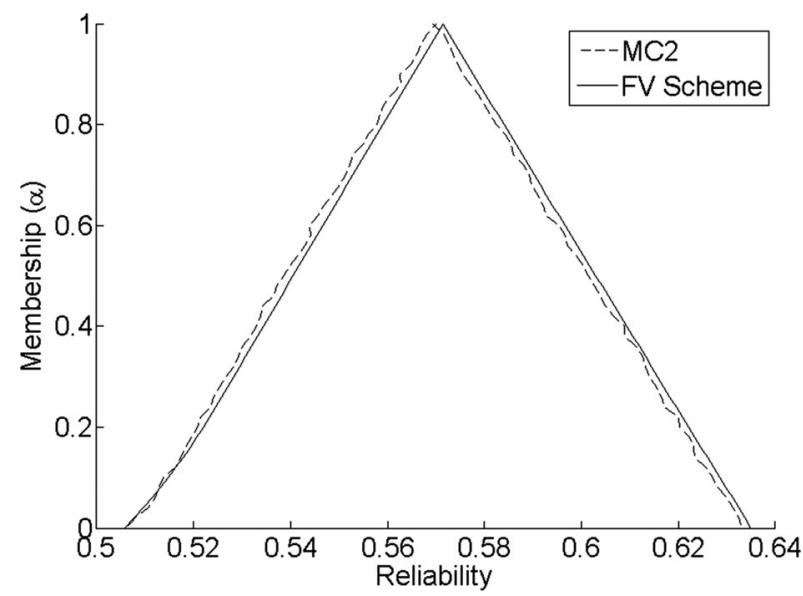

Fig. 10. Membership function of fuzzy reliability $\widetilde{R}(t)$ at mission time $t=$ 800 s obtained by MC2 and FV scheme.

scheme, the state space $\mathbb{R}^{+}$of $D_{b}(t)$ has been divided into an admissible mesh $\mathcal{M}=\cup_{n=0,1,2 \ldots}[n \Delta x,(n+1) \Delta x[$, where $\Delta x=1 \mathrm{e}-8 \mathrm{~m}^{2} / \mathrm{s}$ and the time space $\mathbb{R}^{+}$into small intervals $\mathbb{R}^{+}=\cup_{n=0,1,2, \ldots}[n \Delta t,(n+1) \Delta t[$ by setting the time step $\Delta t=1 \mathrm{~s}$. All the experiments were carried out in MATLAB on a PC with an Intel Core 2 Duo CPU at $1.97 \mathrm{GHz}$ and a RAM of 1.95 GB. The MC simulation method with 105 and 106 replications (named $\mathrm{MC} 1$ and $\mathrm{MC} 2$, respectively), and the proposed FV scheme are applied for the fuzzy reliability assessment of the system. The average computation time of MC1 and MC2 is, respectively, 0.94 and $9.40 \mathrm{~s}$, while that of the FV scheme is $0.20 \mathrm{~s}$. The system reliability decreases more rapidly after around $885 \mathrm{~s}$, because at that time the valve could fail, corresponding to the situation when the pump steps to the state " 1 " very quickly and stays there until the valve fails.

The quantitative comparison of the results over a time horizon $1000 \mathrm{~s}$ is shown in Table III. Compared with the results of MC2, the mean absolute relative difference (MARD) of the results of $\mathrm{MC} 1$ is $0.40 \%$, while that of the results of the FV scheme is $0.17 \%$. It is observed that the results of the FV scheme are closer to those of $\mathrm{MC} 2$, which is more accurate than that of $\mathrm{MC} 1$ because of the larger number of simulations
The results of the fuzzy reliability of the system at cut levels $\alpha=0$ and $\alpha=1$ over a time horizon 1000 s obtained by M-C2 and FV scheme are shown in Fig. 8. The lower bound of the fuzzy reliability of the system at cut level $\alpha=0$ decreases more sharply after around $790 \mathrm{~s}$, earlier than the fuzzy reliability at $\alpha=1$. It is seen that the system fails after around $964 \mathrm{~s}$, because at that time the valve is completely failed. The upper bound of the fuzzy reliability at $\alpha=0$ does not experience a rapid decrease because the valve is mostly functioning over the time horizon.

The membership function of fuzzy reliability $\widetilde{R}(t)$ at mission time $t=800 \mathrm{~s}$ at different cut levels $\alpha \in[0,1]$ obtained by MC simulation methods and FV scheme are illustrated in Figs. 9 and 10 (we have uniformly chosen 51 points in $[0,1]$ with a step equal to 0.02 assigned to $\alpha$ ). The average computation times of MC1 and MC2 are 20.19 and 201.94 s, respectively, while that of FV scheme is $15.91 \mathrm{~s}$.

The quantitative comparison of the results of the membership functions obtained by the MC simulation methods and FV scheme is shown in Table IV. Compared with the results of MC2, the MARD of the results of MC1 is $0.38 \%$, while that of the $\mathrm{FV}$ scheme is $0.27 \%$.

The above results show that the FV scheme achieves comparable results as MC2, with less computational burden.

\section{CONCLUSION}

In system reliability modeling, it is important to be able to describe multiple-dependent degradation processes, while including the uncertainty in their quantitative evaluation. In this study, we have considered the degradation dependence among different system components and within one component in the framework of PDMP modeling. Both PBMs and MSMs are used to describe the components degradation behavior. Epistemic uncertainty due to the incomplete or imprecise knowledge about the degradation processes and the governing parameters is included by describing the model parameters as FNs. For the calculation of the system (fuzzy) reliability, the FV method has been extended and shown to lead to comparable results as MC simulation, but with reduced computing time.

In future research, it will be interesting to consider the situation when aleatory uncertainty is associated with the parameters in the PDMP model. 


\section{ACKNOWLEDGMENT}

The authors would like to thank D. Vasseur, A. Despujols, and E. Ardillon from the Department Industrial Risks Management (MRI), Electricité de France R\&D, for their insightful comments.

\section{REFERENCES}

[1] Y. Wang and H. Pham, "Modeling the dependent competing risks with multiple degradation processes and random shock using time-varying copulas," IEEE Trans. Rel., vol. 61, no. 1, pp. 13-22, Mar. 2012.

[2] J. Lei, F. Qianmei, and D. W. Coit, "Reliability and maintenance modeling for dependent competing failure processes with shifting failure thresholds," IEEE Trans. Rel., vol. 61, no. 4, pp. 932-948, Dec. 2012.

[3] N. Rasmekomen and A. K. Parlikad, "Maintenance optimization for asset systems with dependent performance degradation," IEEE Trans. Rel., vol. 62, no. 2, pp. 362-367, Jun. 2013.

[4] M. Daigle and K. Goebel, "A model-based prognostics approach applied to pneumatic valves," Int. J. Prognostics Health Manage., vol. 2, p. 008, 2011.

[5] M. Daigle and K. Goebel, "Multiple damage progression paths in modelbased prognostics," in Proc. IEEE Aerosp. Conf., 2011, pp. 1-10.

[6] E. Keedy and Q. Feng, "A physics-of-failure based reliability and maintenance modeling framework for stent deployment and operation," Rel. Eng. Syst. Safety, vol. 103, pp. 94-101, 2012.

[7] S. Reggiani, S. Poli, M. Denison, E. Gnani, A. Gnudi, G. Baccarani, S. Pendharkar, and R. Wise, "Physics-based analytical model for HCS degradation in STI-LDMOS transistors," IEEE Trans. Electron Devices, vol. 58, no. 9, pp. 3072-3080, Sep. 2011.

[8] O. Chryssaphinou, N. Limnios, and S. Malefaki, "Multi-state reliability systems under discrete time semi-Markovian hypothesis," IEEE Trans. Rel, vol. 60, no. 1, pp. 80-87, Mar. 2011.

[9] M. Giorgio, M. Guida, and G. Pulcini, "An age-and state-dependent Markov model for degradation processes," IIE Trans., vol. 43, pp. 621-632, 2011.

[10] W. Li and H. Pham, "Reliability modeling of multi-state degraded systems with multi-competing failures and random shocks," IEEE Trans. Rel., vol. 54, no. 2, pp. 297-303, Jun. 2005.

[11] A. Lisnianski and G. Levitin, Multi-State System Reliability: Assessment, Optimization and Applications. Singapore: World Scientific, 2003.

[12] Y.-F. Li, E. Zio, and Y.-H. Lin, "A multistate physics model of component degradation based on stochastic petri nets and simulation," IEEE Trans. Rel., vol. 61, no. 4, pp. 921-931, Dec. 2012.

[13] Y.-H. Lin, Y.-F. Li, and E. Zio, "Dynamic reliability models for multiple dependent competing degradation processes," in Proc. Eur. Safety and Rel. Conf., 2014, pp. 775-782.

[14] P.-E. Labeau, "A Monte Carlo estimation of the marginal distributions in a problem of probabilistic dynamics," Rel. Eng. Syst. Safety, vol. 52, pp. 65-75, 1996.

[15] M. Marseguerra and E. Zio, "Monte Carlo approach to PSA for dynamic process systems," Rel. Eng. Syst. Safety, vol. 52, pp. 227-241, 1996.

[16] R. Eymard and S. Mercier, "Comparison of numerical methods for the assessment of production availability of a hybrid system," Rel. Eng. Syst. Safety, vol. 93, pp. 168-177, 2008.

[17] C. Cocozza-Thivent, R. Eymard, and S. Mercier, "A finite-volume scheme for dynamic reliability models," IMA J. Numerical Anal., vol. 26, pp. 446-471, 2006.

[18] R. Eymard, S. Mercier, and A. Prignet, "An implicit finite volume scheme for a scalar hyperbolic problem with measure data related to piecewise deterministic Markov processes," J. Comput. Appl. Math., vol. 222, pp. 293-323, 2008.

[19] M. Laviolette and J. W. Seaman, Jr., "The efficacy of fuzzy representations of uncertainty," IEEE Trans. Fuzzy Syst., vol. 2, no. 1, pp. 4-15, Feb. 1994.

[20] Y. Ding, M. J. Zuo, A. Lisnianski, and Z. Tian, "Fuzzy multi-state systems: General definitions, and performance assessment," IEEE Trans. Rel., vol. 57, no. 4, pp. 589-594, Dec. 2008.

[21] Y. Liu, W. Tang, and R. Zhao, "Reliability and mean time to failure of unrepairable systems with fuzzy random lifetimes," IEEE Trans. Fuzzy Syst, vol. 15, no. 5, pp. 1009-1026, Oct. 2007.

[22] M. Bazu, "A combined fuzzy-logic and physics-of-failure approach to reliability prediction," IEEE Trans. Rel, vol. 44, no. 2, pp. 237-242, Jun. 1995.
[23] S. A. Sandri, D. Dubois, and H. W. Kalfsbeek, "Elicitation, assessment, and pooling of expert judgments using possibility theory," IEEE Trans. Fuzzy Syst., vol. 3, no. 3, pp. 313-335, Aug. 1995.

[24] Y. Liu, H. Huang, and G. Levitin, "Reliability and performance assessment for fuzzy multi-state elements," Proc. Inst. Mech. Eng, Part O, J. Risk Rel., vol. 222, pp. 675-686, 2008.

[25] Y. Li, E. Zio, and Y.-H. Lin, "A multistate physics model of component degradation based on stochastic petri nets and simulation," IEEE Trans. Rel, vol. 61, no. 4, pp. 921-931, Dec. 2012.

[26] H. Tanaka, L. Fan, F. Lai, and K. Toguchi, "Fault-tree analysis by fuzzy probability," IEEE Trans. Rel, vol. R-32, no. 5, pp. 453-457, Dec. 1983.

[27] D. Singer, "A fuzzy set approach to fault tree and reliability analysis," Fuzzy Sets Syst., vol. 34, pp. 145-155, 1990.

[28] J. Dunyak, I. W. Saad, and D. Wunsch, "A theory of independent fuzzy probability for system reliability," IEEE Trans. Fuzzy Syst, vol. 7, no. 3, pp. 286-294, Jun. 1999.

[29] Y. Ding and A. Lisnianski, "Fuzzy universal generating functions for multi-state system reliability assessment," Fuzzy Sets Syst., vol. 159, pp. 307-324, 2008.

[30] Y. F. Li, Y. Ding, and E. Zio, "Random fuzzy extension of the universal generating function approach for the reliability assessment of multi-state systems under aleatory and epistemic uncertainties," IEEE Trans. Rel., vol. 63, no. 1, pp. 13-25, Mar. 2014.

[31] M. Sallak, W. Schön, and F. Aguirre, "Reliability assessment for multistate systems under uncertainties based on the Dempster-Shafer theory," IIE Trans., vol. 45, pp. 995-1007, Sep. 1, 2012.

[32] C. Baudrit, D. Dubois, and D. Guyonnet, "Joint propagation and exploitation of probabilistic and possibilistic information in risk assessment," IEEE Trans. Fuzzy Syst., vol. 14, no. 5, pp. 593-608, Oct. 2006.

[33] C. Baudrit, D. Dubois, and N. Perrot, "Representing parametric probabilistic models tainted with imprecision," Fuzzy Sets Syst., vol. 159, pp. 1913-1928, 2008.

[34] R. Coudray and J. Mattei, "System reliability: An example of nuclear reactor system analysis," Rel. Eng., vol. 7, pp. 89-121, 1984.

[35] J. Devooght and C. Smidts, "Probabilistic dynamics as a tool for dynamic PSA," Rel. Eng. Syst. Safety, vol. 52, pp. 185-196, 1996.

[36] M. H. Davis, Markov Models and Optimization, Boca Raton, FL, USA CRC Press, 1993.

[37] L. A. Zadeh, "Fuzzy sets," Inf. Control, vol. 8, pp. 338-353, 1965.

[38] L. A. Zadeh, "Fuzzy sets as a basis for a theory of possibility," Fuzzy Sets Syst., vol. 100, pp. 9-34, 1999.

[39] S. Zhang, M. Hodkiewicz, L. Ma, and J. Mathew, "Machinery condition prognosis using multivariate analysis," in Proc. 1st World Congr. Eng. Asset Manage., 2006, pp. 847-854.

[40] P. Moussou, S. Cambier, D. Lachene, S. Longarini, L. Paulhiac, and V. Villouvier, "Vibration investigation of a French PWR power plant piping system caused by cavitating butterfly valves," ASME Publications-PVP, vol. 420, pp. 99-106, 2001.

[41] M. Sallak, C. Simon, and J.-F. Aubry, "A fuzzy probabilistic approach for determining safety integrity level," IEEE Trans. Fuzzy Syst., vol. 16, no. 1, pp. 239-248, Feb. 2008. 